\title{
Un acercamiento a la concepción de gobernabilidad en los discursos presidenciales de José María Figueres Olsen
}

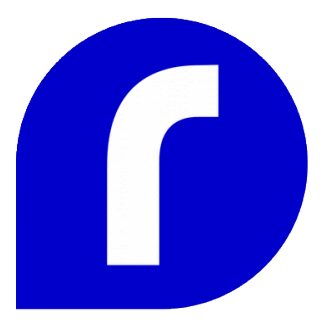

Recibido: 3 de marzo 2016

Revisado: 6 de mayo 2016

Aprobado: 17 de junio 2016

Luis Diego Soto Kiewit Sociólogo costarricense; docente e investigador de la Escuela de Sociología de la Universidad Nacional en Costa Rica. Además, labora como investigador en el Instituto de Estudios Sociales en Población

(IDESPO) de dicha universidad. Entre sus temas de interés se encuentran: imaginarios políticos,

interdisciplinariedad, tecnología e innovación. Correo electrónico: kiwit45@hotmail.com

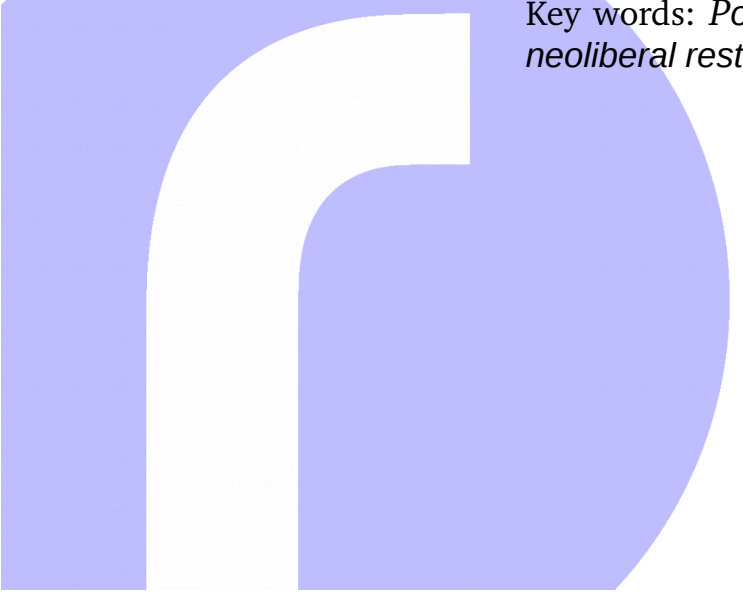

http://investiga.uned.ac.cr/rupturas/ (c) (i) (2)
Resumen: El artículo analiza las alocuciones del expresidente de Costa Rica José María Figueres Olsen en sus discursos de 1 de mayo ante la Asamblea Legislativa, con la finalidad de conocer la concepción de gobernabilidad que se propone en estos. Se expone la relación que existe entre el discurso de gobernabilidad y las políticas orientadas a la transformación del Estado y la institucionalidad pública en Costa Rica. Concomitantemente, exhibe el sentido y la forma en la que el presidente entiende este fenómeno en la realidad nacional.

Palabras clave: discurso político; gobernabilidad; Estado;, análisis crítico del discurso; reestructuración neoliberal

\section{Approach to the Conception of Governability in the Presidential Speeches of José María Figueres Olsen}

Abstract: This paper analyses the speeches given by former president of Costa Rica José María Figueres Olsen on May 1st in front of the Legislative Assembly, in order to know the concept of governability proposed in these. The article focuses on the relationship between the discourse of governability and the policy oriented to the transformation of state and public institutions in Costa Rica, while exposing the sense and the way in which the president understands the phenomenon in the national reality.

Key words: Political Speech, Governability, State, Critical Discourse Analysis, neoliberal restructuring 
Un acercamiento a la concepción de gobernabilidad en los discursos presidenciales de José María Figueres Olsen

Los discursos ejercen el poder porque transportan un saber con el que se nutre la conciencia colectiva individual. Este conocimiento emergente es la base de la acción individual y colectiva, así como el fundamento de la acción formativa que moldea la realidad. (Jäger 2003, 69)

Este artículo constituye una reflexión conexa al trabajo presentando en el artículo: "Concepción de Estado en los discursos de Figueres Olsen: un modelo genérico de reforma", publicado en la Revista Rupturas, vol. 4, número 2.

El autor manifiesta su agradecimiento a Maynor Mora, a Oscar Navarro, a Carlos Madrigal y a José Andrés Díaz, por los diversos aportes y comentarios que realizaron a los borradores de este texto

\section{Introducción}

El presente artículo analiza el discurso sobre la gobernabilidad del expresidente Figueres en relación con el contexto de su Gobierno, lo que implica contestar la pregunta: ¿qué significado tiene que, en los discursos presidenciales, se hable de gobernabilidad en ese momento concreto de la realidad nacional?

Para la resolución de esa interrogante se caracterizó el discurso sobre la gobernabilidad en el marco de relaciones y contingencias del momento de elocución (contexto). Lo anterior permitió establecer vínculos entre causas y situaciones asociadas a la gobernabilidad y la necesidad de transformación del Estado.

Para llevar a cabo esa labor se fijaron relaciones en el nivel discursivo entre esa concepción de gobernabilidad y la discusión sobre la forma del Estado, las cuales se trabajaron desde la propuesta del modelo argumentativo de Toulmin (2003), pues este permitió reconstruir la conexión de las ideas expuestas en las alocuciones analizadas.

\section{Elementos metodológicos y teóricos para el análisis de discurso}

La reflexión sobre la perspectiva de gobernabilidad en los discursos se construyó desde el enfoque de Análisis Crítico de Discurso, pues este posibilita una lectura situada en los planteamientos y las ideas en ellos esbozadas.

El posicionamiento desde ese análisis crítico permite ver que los discursos no son solamente una serie de palabras y frases. Por el contrario, son pronunciamientos con implicaciones reales sobre lo social, sobre el contexto en el cual toman sentido e interactúan. En otras palabras, significa asumir una posición sobre los aspectos sociales, económicos y políticos de la 
realidad o algún tema concreto de la realidad sobre el cual se está hablando, es decir, los discursos son prácticas, son acciones sociales (Van Dijk, 2005).

El hecho de denominar el discurso como acción advierte que toda enunciación tiene una intencionalidad, ya sea manifiesta o no. Así cuando se habla del pronunciamiento del presidente ante la Asamblea Legislativa, se trata de un espacio de rendición de cuentas, un ejercicio para legitimar el accionar del Poder Ejecutivo.

Desde esa perspectiva, los discursos son visualizados como realizaciones estratégicas, en tanto los sujetos, desde un proceso consciente, trabajan en su pronunciamiento; lo hacen coherente, para trasmitir su visión y posición ante aspectos específicos de la realidad. Desde este talante, podemos denotar que esa función legitimadora que puede cumplir en el escenario político los hace parte de las prácticas hegemónicas (Laclau y Mouffe, 1987).

\section{Sobre el procedimiento de trabajo}

La reflexión expuesta se construyó desde el trabajo y análisis de los cuatro discursos presidenciales del primero de mayo ante la Asamblea Legislativa ${ }^{1}$.

El abordaje de los discursos estuvo guiado, primero, por la identificación de los aspectos generales del concepto de gobernabilidad. Luego, siguió la búsqueda y definición de los vínculos que se establecían entre este y las acciones propuestas o sugeridas en el texto del discurso, es decir, la búsqueda de intencionalidades discursivas.

1. Es importante destacar que la presentación de esos discursos es un mandato constitucional que los presidentes deben acatar. Se trata, fundamentalmente, de un ejercicio de rendición de cuentas frente a la Asamblea Legislativa.

De manera más concreta, los dos procedimientos empleados para el análisis, fueron los siguientes: el análisis pragmático del discurso (desde el ACD) y el argumentativo según la propuesta de Toulmin (2003). La articulación de ambos procedimientos tiene un sentido práctico-instrumental, en la que el modelo argumentativo tiene la función de presentar un "mapa" general del argumento de Figueres sobre la gobernabilidad, que sirve para enmarcar y darles sentido a las funciones estratégicas del discurso (del análisis pragmático). La vinculación de la información brindada por ambas herramientas posibilita dar una explicación del sentido de las alocuciones del expresidente, como una expresión de ideas con una intencionalidad en un contexto especifico.

\section{Análisis pragmático del discurso}

Respecto del análisis pragmático del discurso, la centralidad de la discusión y de la reflexión pasa a lo situacional, al sentido que las enunciaciones tienen en un contexto de recepción y de referencia.

En este análisis, el contexto de enunciación se hace el espacio fundamental para el análisis y la comprensión de los discursos, dado que brinda las 
herramientas y las claves que le dan sentido a lo expuesto. El contexto se entiende como la variedad de factores relevantes en la configuración del discurso; acá pueden situarse procesos históricos, políticos, sociales y económicos, a la vez que las condiciones del espacio y los actores en juego.

Siguiendo la idea anterior y según la propuesta dada por Soto (2014), el material se trabajó con la siguiente guía:

1) la identificación de variables y de subvariables para la; 2) búsqueda de estas en el sentido de tres planos: el de presencia (la ubicación en el texto), el de cualidad (sentido que tiene en el texto) y el de la coexistencia (relación y posible determinación que puede tener una sobre otra), para luego; 3) determinar las relaciones contextuales que permitieron el análisis desde la perspectiva teórica.

\section{Análisis argumentativo de Toulmin}

En este otro nivel, de la revisión de la construcción de los argumentos, el abordaje de los discursos se basó en el modelo argumentativo de Stephen Toulmin (2003). Este modelo permitió establecer relaciones y vínculos entre los diversos aspectos constitutivos del discurso, que están orientados a convencer y a posicionar perspectivas de la realidad en las personas (Harauda, 2009), lo que permitió determinar, con mayor claridad, el sentido de lo propuesto, en relación con la idea de gobernabilidad y la discusión sobre el Estado.

La forma y el papel de los argumentos en los discursos apoyan, de manera importante, la visión del autor respecto de la situación o problema. Según Toulmin (2003) en la formalización (tradicional) de los argumentos, se dejan de lado aspectos relevantes que se establecen con la finalidad de persuadir y de convencer, para centrarse en su valía en términos formales. Es ahí donde reside la importancia del estudio y la exposición de las estrategias retóricas presentes en los discursos.

Al igual que se propuso en Soto (2014), el empleo del modelo consistió en ubicar, dentro del texto, los siguientes elementos que conforman la argumentación:

1. Afirmaciones (Claims): son las afirmaciones que el actor del discurso plantea con pretensiones de validez.

2. Respaldos (Grounds): son los datos que, en el discurso, se proporcionan para dar respaldo a la afirmación inicial y por lo general hacen referencia a hechos.

3. Garantías (Warrants): son los principios generales que sirven en la mediación o ligamen entre las afirmaciones y los respaldos.

4. Apoyos (Backings): son los apoyos que permiten dar soporte a los respaldos. 
5. Calificadores modales (Modal qualifiers): son adverbios modales que introducen reservas a la validez en el argumento del autor.

6. Posibles refutaciones (Posible rebuttals): representan las reservas en el argumento del autor; son las que evidencian los posibles casos excepcionales.

Con base en esta estructura, se realizó la búsqueda de la organización argumentativa del discurso; asimismo, se hace la salvedad de que no fue posible ubicar los componentes denominados: calificadores modales y posibles refutaciones o reservas, pues no eran parte de los argumentos presentados en los discursos; su forma de argumentar no daba espacio para excepciones ${ }^{2}$.
2. Estos últimos son más comunes en los argumentos científicos o disciplinares, pues en ellos, se tiende a mostrar
las posibles condiciones de afectación que pueden variar lo que se está planteando o razonando. No pasa así en textos de los discursos estudiados, cuya la intencionalidad es exponer y promover cierto curso de acción, pues significaría la introducción de dudas o

Es relevante señalar que esa adecuación evidencia una limitación del modelo argumentativo Toulmin, pues permite apreciar que en su propuesta no se contemplan esas variaciones contextuales ( $y$ de presentación), las cuales determinan la estructura de los argumentos. Esto sucede porque no todas las alocuciones se conforman de la misma manera ni emplean los mismos recursos para dar soporte a las aserciones. En el caso de los discursos políticos, las formas de argumentación tienen particularidades, por cuanto se deben contemplar adaptaciones que den cuenta del sentido de estos.

El empleo de esta técnica responde a la necesidad tanto de articular en el discurso las variables estudiadas como también de ver la forma argumentativa en que son presentadas, con el fin de convencer, de persuadir y de generar un cambio en el público/auditorio oyente (o lector) ${ }^{3}$. Además, 3. El mensaje se brinda frente a busca evidenciar las estrategias retóricas de legitimación de la necesidad del cambio y de la visión acerca del Estado.

La revisión de la estructura argumentativa permitió el ejercicio reconstrucción del discurso, lo que posibilitó articular la noción de gobernabilidad y las medidas neoliberales pretendidas por el Gobierno.

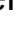 \\ ade} difusión nacional. Por lo tanto, podemos llegar aquí a la conclusión de que su pronunciamiento tiene metas y objetivos, es una enunciación estratégica, con la cual pretende tener un efecto ante su auditorio.

\section{Reconstrucción de la concepción de gobernabilidad en los discursos presidenciales de Figueres Olsen}

En este apartado, se establece, en forma pormenorizada, la noción de gobernabilidad del Presidente Figueres Olsen en sus discursos del primero de mayo. Esta nos permite identificar cuál es la idea que tiene sobre la gobernabilidad, a la vez que da la posibilidad, en un segundo momento, de establecer cuál es el papel que juega ese discurso en el desarrollo de las acciones del Gobierno.

\section{Definición de gobernabilidad}

Esta permite dilucidar la manera en la que Figueres Olsen entiende, o más bien, expone la gobernabilidad en sus discursos. 
A lo largo de los discursos, se identificó una concepción de gobernabilidad que se desagrega en dos distintas. Por un lado en el discurso inicial (1995), se habla de esta en términos de ingobernabilidad y se le define como una capacidad institucional (escasa e insuficiente) para gobernar, como se ve en la siguiente referencia:

Por eso, cuando el conjunto de las instituciones estatales se debilita, muchas de ellas pierden sus fines originales para convertirse en islas burocráticas que hacen poco o que pretenden imponer sus propios fines a la sociedad. Cuando la sociedad va perdiendo respeto y confianza en ellas, surgen y crecen los peligros de la injusticia social, del estancamiento económico y de la desintegración nacional. Cuando eso ocurre, se ingresa en un período de ingobernabilidad, es decir, en un período en el cual la capacidad institucional para gobernar se vuelve cada vez más escasa e insuficiente para enfrentar los retos nacionales con oportunidad y con posibilidades

de éxito (Figueres 1995, 2. El resaltado no pertenece al texto original).

La definición en los términos expuestos, como incapacidad de gobernar, centra el sentido en el espacio de la política (en lo instituido), mientras sobre ella reposa la responsabilidad de haber perdido sus fines originales, lo cual conlleva una pérdida de confianza, y ambas, a la vez, limitan la posibilidad de acción del Gobierno (actor que aparece en última instancia como el detentor del poder de acción).

En la concepción anterior, se ofrece un desplazamiento importante, pues hay una dilución de la responsabilidad de la figura del Gobierno (actores) hacia la institucionalidad en general, pues esta tiene sentido en un espacio anterior y más amplio: el Estado. Esto se puede vislumbrar en la diferenciación que hace para referirse a una institucionalidad pública en la cual no incluye al Gobierno (actores); por el contrario, los aparta y los pone en el lugar de la afectación. En consecuencia, se ven limitados para enfrentar los retos.

Acá hay un punto por destacar en la referencia anterior, el discurso está cumpliendo la función estratégica de la deslegitimación de las condiciones de la institucionalidad, al asignar calificativos peyorativos de desgastada o isla burocrática.

Por otro lado, en la segunda concepción de gobernabilidad, presente en los discursos, hay una transición, en tanto la expone como la capacidad de la 
sociedad y del Gobierno de satisfacer las necesidades y demandas de las mayorías.

La variante que se sugiere, en esta nueva concepción, hace referencia a que la condición que determina la gobernabilidad, ingobernabilidad o crisis de gobernabilidad es la capacidad de satisfacción de las necesidades y demandas de las mayorías. También, se introduce un segundo actor, la sociedad. Como se puede ver en Figueres $(1996,2)$ :

En mi informe ante la nación del año pasado, me referí a

la crisis de gobernabilidad que el país había experimentado en los últimos tiempos. De manera resumida, podemos describirla como una creciente falta de capacidad de la sociedad y de sus instituciones para satisfacer las necesidades de las mayorías, y para abrirse paso de forma unida y solidaria, hacia un futuro de mayor bienestar (El resaltado no pertenece al texto original).

En esa otra concepción se plantea que hay un desplazamiento; pasa de centrarse en la forma de generar las condiciones para la gobernabilidad, desde una perspectiva de la vinculación sociedad-Gobierno, a la idea de la posibilidad de desarrollar acciones orientadas a generar bienestar. Esto sugiere que la gobernabilidad se justifica en tanto busque propiciar, desde la perspectiva del actor, condiciones de bienestar para la población. Asimismo, trae a colación lo que esboza Roitman (2001): que la idea de gobernabilidad, en tanto razón de Estado, siempre toma sentido en relación con el proyecto de este al que responde, es decir, se denomina gobernabilidad a las condiciones favorables para el accionar del Gobierno y se le justifica con la supuesta búsqueda de bienestar para la población.

La vinculación que se hace de la sociedad como actor determinante, en la gobernabilidad, es consecuente con el discurso de la concertación y la participación que aparece a lo largo de los textos. Según planteaba Roitman (2001), son formas de institucionalizar el conflicto, de disipar y de legitimar las acciones del Gobierno, que siempre están presentes en los discursos de gobernabilidad.

En el fondo lo que se busca es la construcción de hegemonía, pues al contextualizar esas concepciones, se da cuenta de la relación que existe entre esgrimir cuestionamientos a la institucionalidad actual (propia de otras formas de Estado) y justificar el accionar y las políticas que está promoviendo el Gobierno. 


\section{Las dimensiones de la gobernabilidad}

Las dimensiones de la gobernabilidad refieren a los conceptos relacionados con esta, pues en el proceso de análisis de los diversos estudios sobre gobernabilidad (Camou 2001; Ordóñez 2009; Suárez 2002; Tomassini 1996) se constató que esta siempre va acompañada de la presencia de ciertas nociones. Entre esas dimensiones, se encuentran: la eficacia, la legitimidad y la estabilidad, las cuales, a su vez, pueden ser asumidas y visualizadas de diversa forma. Por lo tanto, se justifica la necesidad de hacer revisión de estas en los textos.

\section{Eficacia/Eficiencia}

La primera está centrada en el cumplimiento y en la concreción de los objetivos, mientras la segunda se enfoca en la mejor forma de hacerlo; se podría decir, con la menor cantidad de tiempo y de recursos posibles. Estas primeras dimensiones están relacionadas con la razón del Estado, en tanto ejercicio del poder.

Por un lado, la idea de eficiencia en el marco del discurso presidencial va ligada al fenómeno de crisis institucional, ya que según se plantea, la eficiencia se ve limitada por esta última. Esto dificulta el ejercicio de gobierno, la toma de decisiones y la concreción de las políticas diseñadas por este para sacar avante las metas propuestas en las diferentes áreas: social, económica y ambiental. La eficiencia trata de brindar, de la mejor forma, los servicios que se pueden desarrollar con los recursos disponibles, como se hace visible en Figueres (1996):

[...] La otra causa de la insuficiencia de gobernabilidad es el proceso de deterioro de las instituciones públicas, que todos sentimos en carne propia conforme los servicios públicos pierden calidad y le brindan menos beneficios a la población de los que podrían darle con los recursos disponibles, y conforme las leyes y los procesos administrativos se complicaron, se desactualizaron, y se convirtieron en una camisa de fuerza, cada vez más estrecha, que limita la acción del gobierno, y hace, cada vez, más difícil la toma oportuna de decisiones (2-3).

Por otro lado, la eficacia refiere a la posibilidad de concretar los objetivos previamente formulados (las metas con las cuales el Gobierno inicia su gestión), frente a lo cual en el discurso se plantea que no es posible desarrollarlos dadas las circunstancias. En relación con esto, en la cita anterior aparecen dos figuras importantes: el deterioro de las instituciones 
públicas y las leyes y los procesos administrativos complicados y desactualizados. Lo anterior refleja, nuevamente, el ejercicio orientado a la deslegitimación discursiva de la condición actual de las instituciones y leyes del Estado.

Existe una importante relación entre las transformaciones que el Gobierno pretende desarrollar y las posibilidades de que se alcance esa meta, es decir, la de conferir eficacia al Estado. Así se explica en Figueres (1997):

Perseguimos un Estado más democrático y más eficaz, que plantee sus acciones con la gente y no al margen de la gente. Perseguimos también un país más próspero, que sepa aprovechar de forma inteligente los recursos humanos y naturales de esta zona (10).

Aparece acá legitimando la idea de la eficacia en la función pública; además, la explícita como finalidad de su accionar.

La eficacia y la eficiencia desde la concepción de gobernabilidad que se hace presente en los discursos refiere, por un lado, a la posibilidad de que el Gobierno pueda desarrollar las políticas y las transformaciones que se ven como necesarias para mejorar las condiciones en las instituciones y el Estado; y por otro, que estas tengan efectos positivos en la consecución de los objetivos del Gobierno.

Los discursos, desde esta dimensión de la gobernabilidad, cuestionan y deslegitiman un tipo de accionar en función de otro, se rebaten ciertas características y cursos de acción y se realzan otros. Este accionar está orientado al fomento y a la creación de las condiciones para la institución de cierto orden, de una forma preconcebida de la política.

\section{Legitimidad}

En cuanto a la noción de legitimidad, se establece la dimensión ética del ejercicio político, con la idea del "buen gobierno", razón por la cual el sustento material de esta concepción viene dado por la justicia y la legitimidad como características necesarias de la relación entre Gobierno y sociedad civil.

El Gobierno es visualizado como el encargado de la representación de intereses, de la concreción de las tareas inherentes de generación de bienestar a la población.

La concepción de la legitimidad aparece, en el marco del discurso, asociada; por un lado, a una crisis de relacionamiento social, materializada en la crisis de solidaridad. Y por otro, a una incapacidad de la institucionalidad de legitimarse mediante la práctica, dado que no pueden responder a los 
cambios que los tiempos exigen, al contexto diferenciado que representa la globalización (Figueres, 1995).

El rol de la ciudadanía en la legitimidad del accionar del Gobierno es bien clarificada, cuando en Figueres $(1995,3)$ se define: "La legitimidad del gobierno la otorgan los ciudadanos", lo que le asigna un sentido valorativo por parte de la población al accionar de la institucionalidad pública.

El estado de la legitimidad se asocia, en el discurso, a esa capacidad de gobernar. No obstante, también es presentado como un fenómeno que, en una situación contraria, de deslegitimación, condiciona las posibilidades de sostener y de fortalecer la democracia, como se puede ver en Figueres (1995, 3-4):

La erosión que durante muchos años viene experimentando la capacidad de gobernar, ha avanzado a la par de un fenómeno peligroso para una sociedad que pretende mantener y fortalecer su democracia. Me refiero a la pérdida de legitimidad de las instituciones públicas ante la población. Conforme pierden calidad los servicios públicos, conforme el Estado pierde capacidad para satisfacer las necesidades básicas de las mayorías, la población va perdiendo confianza en el interés y en la capacidad del Estado para resolver sus problemas. $Y$ a la vez, conforme las instituciones públicas pierden legitimidad, el clima nacional se vuelve cada vez más inapropiado para que las acciones gubernamentales rindan frutos que beneficien a todos (El resaltado no pertenece al texto original).

La legitimidad aparece condicionada a la posibilidad de desarrollar las transformaciones, que, desde su perspectiva del Estado, se hacen necesarias, pues este, en términos del ser, no puede responder a esos requerimientos de la población y del contexto.

La legitimidad es una condición necesaria para el desarrollo de los cambios, pues en Figueres $(1997,4)$ se expresa que: "En los países democráticos, la acción de los gobiernos no puede superar el ritmo de cambio al que la sociedad, en su conjunto, ha resuelto someterse". Lo anterior permite comprender el constante interés que se presenta en los discursos para generar un amplio apoyo social bajo procesos de concertación, pues la 
intencionalidad es desarrollar y aplicar las transformaciones. El interés se limita a un sentido pragmático, de crear las condiciones para esos cambios.

\section{Estabilidad}

La noción de estabilidad es uno de los aspectos que se pueden exhibir como punto nodal de la exposición, pues en el discurso se lee la noción de un estado ideal, al cual se quiere llegar producto de una serie de cambios. Acá toma sentido la fuerte presencia de la idea de los equilibrios en los discursos de Figueres Olsen.

La idea de los equilibrios permite hablar de la necesidad de acciones específicas que traigan estabilidad en las diversas áreas (económica, política, social y ambiental). Así, las políticas planteadas y desarrolladas por el Gobierno se pueden justificar, mientras que, desde su perspectiva, son tendentes a traer ese tan importante estado de armonía, el cual por lo común está íntimamente relacionado a la conformación de un Estado diferente. Lo anterior se asocia a los cambios estructurales fundamentales para la consecución de esos equilibrios, como lo menciona Figueres: "Lejos de conformarnos con administrar las crisis cotidianas, hemos buscado reconstituir los grandes equilibrios sociales, económicos y ambientales que hacen posible la sostenibilidad del desarrollo" $(1995,4)$.

A partir de la idea de estabilidad, se premia la capacidad de equilibrio o la habilidad institucional de adaptación a los cambios que se generan en las nuevas condiciones del contexto. Asimismo, se argumenta que la institucionalidad actual ya no responde al nuevo escenario, porque no cumple con las dos condiciones anteriores: eficacia y legitimidad, a causa de que no puede responder acorde a los requerimientos de los nuevos tiempos. Estos son los razonamientos que se hacen presentes en la enunciación de Figueres Olsen y que constituyen el punto crucial desde el cual se justifica ese Estado requerido.

La idea de estabilidad cumple la función, en términos de estrategias discursivas, de deslegitimar supuestos estados de desequilibrios y de legitimar las acciones orientadas a la reconstitución de los equilibrios.

\footnotetext{
Causas de la ingobernabilidad, crisis de gobernabilidad o déficit de 4. Acá se realiza la acotación gobernabilidad $^{4}$ de que si bien el título hace la diferenciación, a lo largo de ese texto se utilizarán como sinónimos, de forma indistinta. Esto con la finalidad de lograr una mejor exposición de las ideas y a sabiendas de que esta decisión no afecta la información presentada, porque las causas presentadas en los discursos son las De manera general, se refiere a las causas de la ingobernabilidad, como aquellos fenómenos (Figura 1) que se manifiestan en la sociedad mismas, lo que no varía es su grado de profundidad.
} 
costarricense y que condicionan o dificultan el accionar de Gobierno, como se verá en los puntos sucesivos.

Figura 1

Causa de la ingobernabilidad

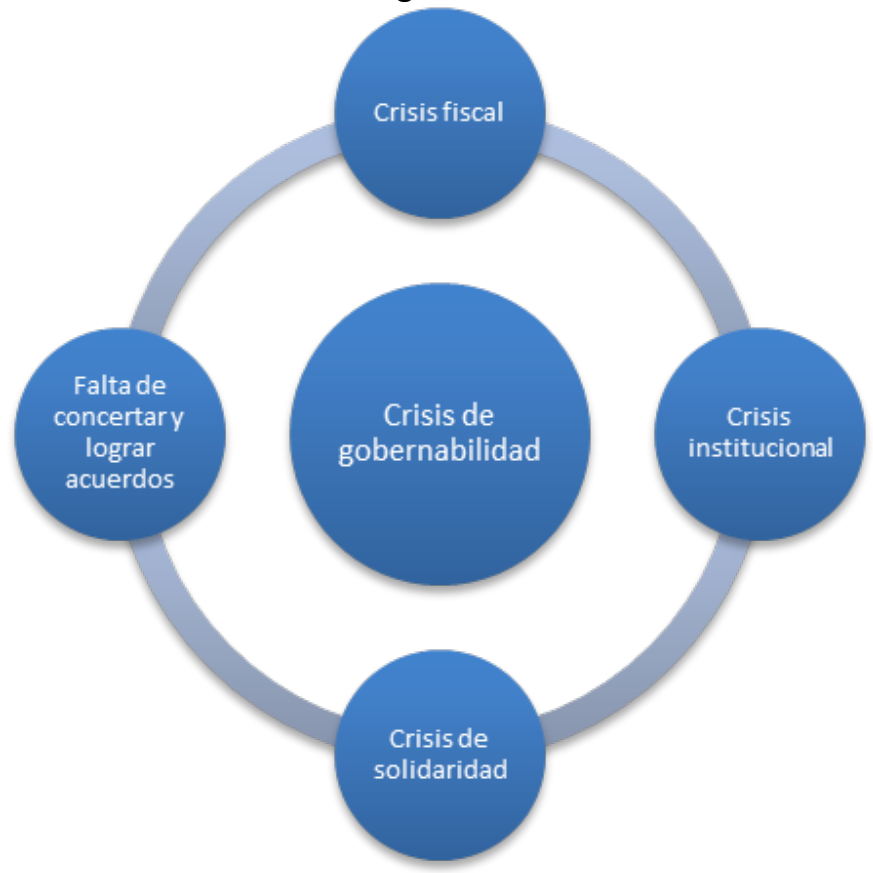

Elaboración propia a partir de los discursos analizados, 2016

5. Por crisis fiscal, se refiere a un aumento importante en el indicador del déficit fiscal, que se entiende como la condición de aumento del gasto público con mayor rapidez que los ingresos tributarios recaudados por el Gobierno.

\section{Crisis fiscal}

La crisis fiscal ${ }^{5}$ aparece como uno de los fenómenos que condicionan el ejercicio del Gobierno y sus posibilidades reales para desarrollar las acciones necesarias para afrontar los problemas nacionales, por tanto de la gobernabilidad.

En los discursos, el déficit fiscal y las condiciones asociadas a este son las áreas centrales para la transformación pues plantea que, al constituir una parte muy elevada del gasto público, dificulta el accionar del Gobierno.

El déficit que se plantea ha aumentado en los últimos años de forma importante por el exceso de gastos de la institución pública. Ese problema aparece constantemente relacionado con la forma de interacción que fomentaba la configuración anterior del Estado; además, obedece a una de las preocupaciones centrales del ajuste estructural. Lo anterior permite visualizar la articulación legitimadora que aparece en los discursos, entre una actividad que causa esas condiciones de ingobernabilidad y una medida fundamental para la consecución del ajuste estructural. 
Los efectos de ese déficit fiscal, según Figueres (1995), pasan por las tres dimensiones de la gobernabilidad, pues tienen que ver con el despilfarro y el desaprovechamiento de los recursos que representa la ineficiencia e ineficacia; también, se vincula a la legitimidad en tanto limita las posibilidades de acción del Gobierno, lo que tiene efectos sobre la confianza de la población. De igual manera, es primordial que, desde la concepción de estabilidad, el déficit fiscal representa la ruptura de los equilibrios en la economía pública, que conlleva a su vez desequilibrios en otras áreas.

La explicación que Figueres propugna en sus discursos sobre este problema, se orienta a dos condiciones, una estructural y otra cíclica. La primera se enfoca en la instauración de un entramado de instituciones que generan un exceso de gastos, a la vez que se halla imposibilitada a cumplir las funciones de cobro y de recaudación. La segunda, a los ciclos electorales, según los cuales las administraciones, en el último año de su gobierno, aumentan sus gastos en algunas áreas estratégicas con la finalidad de influir en las elecciones (Figueres, 1995).

\section{Crisis institucional}

La crisis institucional representa uno de los fenómenos a los que se enfrenta el Gobierno y que condiciona su accionar, por ende, la relación que supone la gobernabilidad, como lo expresa Figueres:

\section{[...] La otra causa de la insuficiencia de gobernabilidad es el proceso de deterioro de las} instituciones públicas, que todos sentimos en carne propia conforme los servicios públicos pierden calidad y le brindan menos beneficios a la población de los que podrían darle con los recursos disponibles, y conforme las leyes y los procesos administrativos se complicaron, se desactualizaron, y se convirtieron en una camisa de fuerza, cada vez más estrecha, que limita la acción del gobierno, y hace, cada vez, más difícil la toma oportuna de decisiones $(1996,2-3$. El resaltado no pertenece al texto original).

La exhibición de la crisis institucional, como una de las condicionantes de la gobernabilidad, justifica y hace necesario (desde la perspectiva del autor) la elaboración de políticas para la transformación de la institucionalidad. Estas acciones son parte de un cambio en el estilo de desarrollo que es propio de ese modelo de "Estado obsoleto" (El ser del Estado). (Figueres 1995, 2), 
pues la condición de esas instituciones como "enfermas, desgastadas y obsoletas" (Figueres 1997, 3) es una situación propia de ese ser del Estado en el contexto inmediato del Gobierno.

La disputa que se presenta en los discursos refiere, de forma clara, al contexto de evocación, a las disputas entre sectores hegemónicos y contrahegemónicos por instituir una formación hegemónica que genere las condiciones propicias para la conformación de su noción de Estado (Laclau y Mouffe 1987).

La crisis en la institucionalidad también tiene efectos directos sobre las tres dimensiones de la gobernabilidad planteadas en el punto anterior. En términos de eficacia, esta crisis es la circunstancia central de las limitaciones que presenta la institucionalidad. En cuanto a la legitimidad, esa imposibilidad de las instituciones de llevar a cabo los fines por los que fueron creadas genera el escenario para que la población pierda la confianza en su accionar, pues no responden a los intereses de los diversos sectores. Al no poder responder a los signos de los tiempos (a las circunstancias que el contexto impone), los equilibrios en las distintas áreas se ven afectados. por eso se plantea la necesidad de reconstruir estos equilibrios, para acceder a esa condición de desarrollo.

Las instituciones cobran así un doble sentido: son a la vez problema y solución. En el escenario del modelo actual (del contexto de los discursos), son claramente una limitante importante a la acción del Gobierno, pero su transformación puede generar las condiciones necesarias para solucionar los problemas que aquejan al país (Figueres 1997).

\section{Crisis de solidaridad}

La crisis de solidaridad aparece en los discursos como otra de las causas de la situación de ingobernabilidad, dado que dificulta el apoyo y la concreción de los acuerdos necesarios. Una de las contingencias que trae al gobierno la crisis de solidaridad, es la falta de disposición a lograr esos acuerdos, la cual Figueres achaca a que

[...] creció el individualismo, el gremialismo y el irrespeto

a los derechos de los semejantes. Se debilitó la disposición a concertar acuerdos, y se fortaleció la intolerancia y la rigidez ante los intereses de los demás y ante los intereses de toda la colectividad. Se debilitó el espíritu de lucha, y surgió en cambio la evasión ante los peligros y los retos nacionales y la búsqueda de soluciones mágicas e ilusorias $(1995,3)$. 
Uno de los factores que se introduce en la referencia antepuesta es la oposición hacia los intereses de los demás por los propios, como una de las razones de ese individualismo. Esto redimensiona la relación y causa oposición a las medidas del Gobierno, en tanto orientadas al bien común.

Esa tercera crisis (de solidaridad) corresponde a la imposibilidad de desarrollar las transformaciones necesarias, pues fue evidenciada cuando se intentaron aplicar medidas de ajuste (Figueres 1997, 2-3). Esa perspectiva deja la oposición en el plano de simples acciones egoístas e individualistas, desapareciendo el sentido antagónico y confrontativo que tienen las diversas muestras de divergencia a las medidas de ajuste.

Lo anterior se llena de sentido, pues la falta de una base de apoyo es la principal limitante de las transformaciones. Por lo cual el llamamiento a la solidaridad y a la participación parece (a nivel discursivo) un sentido legitimador y hegemónico orientado a propiciar sustento y aceptación a las medidas del Gobierno. Incluso, al realizar el cruce con la referencia de los actores instituidos e incluidos en las negociaciones efectuadas, se da cuenta de esto, pues son actores estratégicos, con una capacidad de injerencia sobre las decisiones tomadas en espacios como la Asamblea Legislativa. Por ejemplo, la negociación que representó el Pacto Figueres-Calderón, la cual permitió la aprobación de algunos de los proyectos del Gobierno de Figueres, a saber: el tercer Programa de Ajuste Estructural y los proyectos de modernización del sistema financiero nacional.

En la escena expuesta, se reafirman los cuestionamientos hacia el sentido de la democracia delegativa (O'Donnell 1994), pues los procedimientos de legitimación que esta brinda no dan el margen de acción necesario para poder desarrollar políticas orientadas al cambio.

\section{Falta de concertar y lograr acuerdos}

Con esa crítica a las posibilidades articulatorias y de legitimación que la democracia delegativa asigna, aparece la figura de la concertación y de la generación de espacios para el logro de acuerdos, lo cuales se ven afectados por la crisis de solidaridad.

Como partes integrantes de las limitaciones para lograr acuerdos por medio de la concertación, se mencionan dos aspectos: primero, la falta de compromiso por el interés general (Figueres 1997, 3), que a lo largo de los discursos aparece relacionada a la figura de los partidos políticos y los actores a lo interno de estos. Segundo, el exacerbado temor a los cambios (Figueres 1997, 3).

En cuanto al temor a los cambios, en Figueres se amplia:

Sabemos, además, que los cambios suelen provocar

temores e incertidumbres, y que impulsarlos en muchos

campos de la sociedad durante un corto período de 
tiempo, tiende a incrementar esa natural resistencia de toda sociedad humana a modificar sus formas de organizarse y de convivir. Mis compañeros del equipo de Gobierno y yo, recibimos muchas veces los consejos de algunos que nos recomendaban reducir el ritmo y la cantidad de las transformaciones y basarnos más en las imágenes propagandísticas positivas; pero el país no estaba para postergar la solución de sus necesidades urgentes con tal de mejorar la imagen del Gobierno. No fue para eso que nos presentamos a las elecciones. Por eso escogimos la estrategia que, a nuestro modo de ver las cosas, era la que correspondía para el momento por el cual pasaba el país $(1998,3)$.

Como se puede observar, ante el amplio número de transformaciones y lo profundo de las mismas, se advierte la gran resistencia que iban a generar por parte de los diversos sectores sociales. En este sentido el miedo a los cambios, como figura discursiva, tiene una función reificadora (Lukács, 1969), ya que funge como un naturalizador de la resistencia a los cambios, no como conflicto, si no como simple resistencia instintiva a condiciones diferenciadas, lo que desvirtúa los antagonismos y los deslegitima de previo, pues los califica como una acción irracional.

Acá discurre, de forma importante, el discurso del beneficio general, con la finalidad de invitar a deponer el interés particular de los diversos sectores sociales, como una forma de concretar los acuerdos.

En ese sentido, se achacan las limitaciones al avance del Gobierno a las manifestaciones o a los síntomas del deterioro moral, que Figueres y su equipo de trabajo visualizan en la sociedad costarricense, como se expresa en Figueres:

[...] pienso que ciertos síntomas de deterioro moral que hoy percibimos son una etapa pasajera en la vida nacional, y que pronto vendrán tiempos en los que la nacionalidad costarricense experimentará un fortalecimiento de sus fibras espirituales. Pero mientras eso no ocurra, la acción del gobierno se ve obstaculizada por la pérdida de solidaridad de los más fuertes con los 
más débiles, por la reticencia de unos grupos a hacer concesiones en sus intereses de corto plazo en beneficio del interés nacional de largo plazo, por las actitudes evasivas ante las responsabilidades de los individuos y los grupos con la sociedad (1995, 3).

Otra forma de desvirtuar el antagonismo y la resistencia (condición inherente de lo político) es apelando a un criterio moral, de un desinterés por la condiciones de los demás por la búsqueda de un beneficio propio. Esto se une con la falta de valores espirituales de ayuda al prójimo y de respeto, condiciones necesarias (desde su perspectiva) para enfrentar los grandes retos que se le presentan al país.

Otra de las manifestaciones del deterioro moral en la sociedad, según el presidente, es lo que él denomina la confrontación innecesaria, como lo expone Figueres:

El deterioro de nuestros valores básicos se expresa cuando ciertos dirigentes gremiales se separan de los intereses de los grupos que representan para promover los suyos propios, y llegan a preferir la confrontación innecesaria con el gobierno en lugar del diálogo franco y provechoso $(1995,3)$.

De esta manera, se asume que las acciones que no sean canalizadas por la vía institucionalizada o propiciadas por el Gobierno son parte de esa confrontación innecesaria al accionar del Gobierno; por lo tanto, las huelgas, las movilizaciones y las protestas son causantes de crisis de gobernabilidad.

A lo largo de los discursos, los fenómenos o situaciones que se asocian a la ingobernabilidad dificultan las acciones y las decisiones del Gobierno, ya sea que vengan de la ciudadanía o de las condiciones de la institucionalidad.

En cuanto a los espacios en los cuales aparecen las causas de la crisis de gobernabilidad, se puede encontrar, en apariencia, un entrecruzamiento entre las que se desarrollan dentro del espacio del Gobierno y la institucionalidad (crisis institucional y crisis fiscal) y las que toman cuerpo en un espectro más amplio, el de lo político, como son las siguientes: la falta de concreción de acuerdos y la falta de solidaridad. 


\section{Transformaciones en la forma de Estado y gobernabilidad: la legitimación de un curso de acción}

Como se ha venido mostrando a lo largo de los distintos apartados, la gobernabilidad como herramienta discursiva cumple una doble función en los textos: de condicionante y de justificante de las transformaciones.

El primero de los argumentos (Figura 2) muestra una de esas funciones, la que se relaciona con el condicionamiento que significa la ingobernabilidad para poder instituir otra forma de Estado y que el Gobierno pueda accionar ante las circunstancias. Según se esboza, el accionar del Gobierno se ve afectado por la ingobernabilidad; por lo tanto, este se encuentra obstaculizado para desarrollar las transformaciones requeridas.

El argumento indica que el Gobierno es afectado por la ingobernabilidad o crisis de gobernabilidad, lo cual se refleja en las circunstancias citadas a continuación:

- La falta de legitimidad que crea un clima inapropiado para las acciones gubernamentales. Esto porque las instituciones son ineficientes e ineficaces, por ende, pierden la confianza y la legitimidad.

- El marco jurídico y administrativo que dificulta desarrollar acciones necesarias (transformaciones), porque su desactualización trae problemas de eficacia y eficiencia.

- Aumento del déficit, que constriñe las posibilidades de accionar del Gobierno.

- El aumento del individualismo y del gremialismo que dificulta los acuerdos, que en una situación de crisis son fundamentales.

El nodo de este argumento está puesto en los factores que condicionan la legitimidad de las acciones del Gobierno y la crisis de solidaridad. Ambos son propuestos como los principales limitantes de la gobernabilidad, que impiden que el Gobierno pueda gestionar las transformaciones.

Los datos que el autor presenta para sustentar su argumento (Garantías), pasan por diversas contingencias que impone ese fenómeno de la gobernabilidad limitando su accionar, como son: la falta de legitimidad, un exacerbado individualismo, el problema de ineficiencia e ineficacia (que traen desactualización institucional, jurídica y déficit fiscal).

En cuanto a los refuerzos a sus argumentos (R), encontramos que todos se materializan en una serie de crisis (institucional, en las finanzas públicas y la solidaridad). En Soto (2014), estas se presentaron como las causantes de la inviabilidad de la forma actual del Estado (el ser), y a la vez, crean un escenario de vicisitudes que dificultan la concreción de transformaciones necesarias para la instauración del deber ser del Estado. Las transformaciones aparecen justificadas por la necesidad de generar un escenario de gobernabilidad que permita al Gobierno responder a las necesidades de la población. 
Figura 2

Modelo de Toulmin

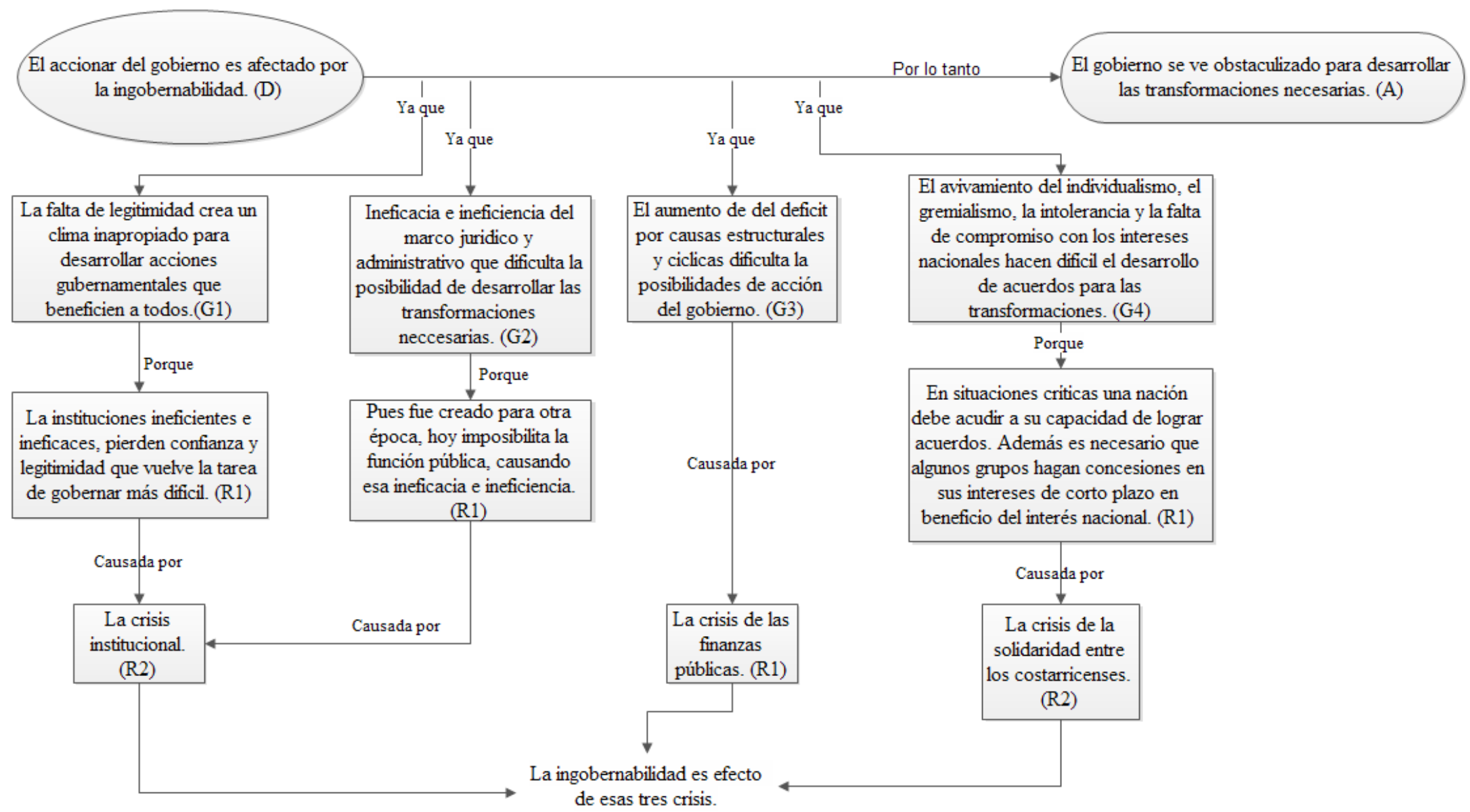

Elaboración propia a partir de los discursos analizados, 2016 
Figura 3

Modelo de Toulmin

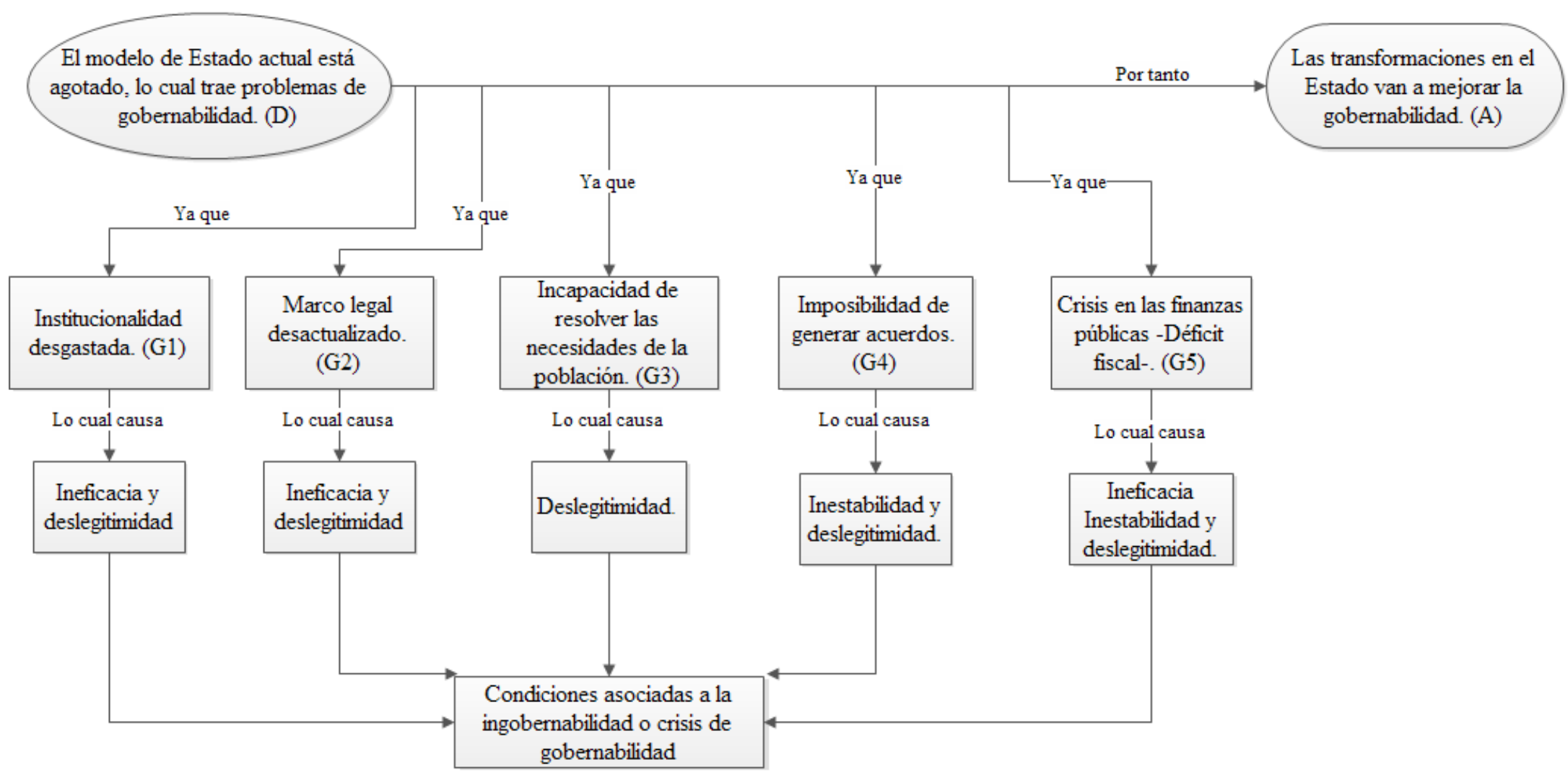

Elaboración propia a partir de los discursos analizados, 2016 
En cuanto al segundo argumento (Figura 3), refiere a los efectos que tiene ese Estado agotado sobre la condición de gobernabilidad, lo que nos presenta la segunda función, la justificación de los cambios. Esta última se expone de la siguiente manera: el modelo de Estado actual está agotado, lo que trae problemas de ingobernabilidad; por lo tanto, las transformaciones que se den en este mejorarán ese escenario de ingobernabilidad.

Las garantías (G) que el autor liga a esa afirmación inicial refieren a los cuestionamientos que, en forma general hace al ser del Estado, son las siguientes: la institucionalidad desgastada, el marco legal desactualizado, la incapacidad de resolver problemas y necesidades de la población, las crisis en las finanzas y la imposibilidad de generar acuerdos, como se puede apreciar a la largo de la reflexión propuesta en Soto (2014).

En cuanto a los refuerzos proporcionados (R), son en su totalidad efectos que tiene la condición del Estado, sobre lo que se denominó dimensiones de la gobernabilidad, las cuales, para este argumento, serían más bien dimensiones de la ingobernabilidad.

El contexto en el que se enmarcan los discursos del presidente Figueres Olsen se puede catalogar como un momento convulso del escenario político nacional, pues como ya se planteó en Soto (2014), hay un conflicto abierto a causa de la conformación de una u otra concepción de Estado. Como lo clarifica Hernández (1996), haciendo alusión a otro fenómeno, que tiene lugar en el mismo escenario:

La coyuntura en la que se dio el acuerdo entre Figueres y Calderón se caracterizó por múltiples conflictos; encuentros y desencuentros entre distintas fuerzas y sectores políticos: entre el Gobierno y la oposición mayoritaria se había verificado un enfrentamiento verbal y práctico, a niveles de la Asamblea Legislativa, por diferencias en cuanto al contenido y tramitación de algunos proyectos; el gobierno había sido objeto de múltiples críticas incluso provenientes del mismo PLN (41-42).

A diferencia de Hernández, en el presente trabajo, se plantea que esos enfrentamientos transcienden el espacio de la política (de lo instituido), para ampliarse al espacio de lo político, como una pugna que si bien se quiere orientar y tramitar por ese espacio de la institucionalidad, toma fuerza en diversas manifestaciones de oposición y de resistencia en un ámbito más general. 
Los discursos analizados son parte del conflicto, implican el posicionamiento del autor respecto de ese contexto de antagonismos y confrontación, aunque su postura esté orientada a disiparlos e instituirlos. En lo referente al poder, si bien la investidura dada por la posición de Figueres Olsen le confiere cuota de legitimidad y de poder (le permite el espacio y posibilidad de comunicar sobre la realidad nacional, las condiciones del país y del Estado), es necesario tomar en cuenta que los discursos son una forma de construcción y de ejercicio de poder. Estos se enmarcan en un escenario de constitución de hegemonía, pues como se reconoce, el poder acá no es fundacional si no se construye en el accionar, y los discursos son parte de este.

Como ya se bosquejó en los apartados anteriores, el lugar desde donde Figueres da sentido a sus argumentos no es neutro; implica una visión ideológica de la realidad, que para el caso específico, en muchas de sus propuestas, refleja cercanía con el ideario neoliberal, como se expuso en Soto (2014).

Ese posicionamiento respecto de la realidad y de la institucionalidad se entiende como una práctica de constitución hegemónica, pues ese escenario y los conflictos que en él se decantan son parte de luchas antagónicas por la conformación de formaciones hegemónicas Laclau y Mouffe (1987).

Sobre la coyuntura de los discursos, Hernández amplía:

Esta conflictividad, de carácter coyuntural, es expresión de una conflictividad estructural que remite a los conflictos generados desde la década pasada [años ochenta] por el proceso de ajuste estructural. Este ha implicado, entre otras cosas, una redefinición del papel del Estado y de sus dimensiones; una estructuración productiva con miras al mercado externo y una apertura al mercado mundial $(1996,42)$.

Así, la gobernabilidad en ese escenario cumple con esa doble funcionalidad; en la legitimación de cierto curso de acción, es un discurso orientando a justificar las acciones y los proyectos del Gobierno, en el sentido de redefinir el papel del Estado y los ámbitos de acción de este.

Desde la lectura propuesta en el presente artículo, la gobernabilidad, como concepto, es parte de un discurso funcional a los intereses instituyentes a la razón del Estado que se quiere posicionar en la realidad. Por lo tanto, no es un fenómeno de la realidad, pues la relación que sugiere es históricamente constituida como conflictiva (en la medida que hay un enfrentamiento constante de concepciones diversas de la realidad), como reflejo de esa condición inherente de lo político. 
Para el caso concreto de los discursos, la concepción de ingobernabilidad, como la escaza o insuficiente capacidad institucional para gobernar, remite a que esta se deba (en su sentido negativo) a toda acción que interfiera el ejercicio de Gobierno, es decir, cualquier condición (institucional o social) que limite la aplicación de las políticas que desde el Gobierno se ven como necesarias.

La segunda concepción de gobernabilidad que brindan los discursos sigue la misma línea anterior, pues si bien hay una variación en términos de la condición que asigna el estado de gobernabilidad o de ingobernabilidad, ya que pasa a ser la posibilidad de satisfacer las necesidades de la mayoría y de abrirse paso hacia un futuro de mayor bienestar, se cambia el tono y modera la posición. No obstante, en el fondo sigue guardando una relación con la posibilidad del accionar y las transformaciones deseadas, mientras tanto la concreción de estas, en última instancia, es lo que permitirá o no brindará ese mayor bienestar.

En el escenario de ingobernabilidad en cual se enmarca el Gobierno, la vía de salida que presenta es la transformación estructural del Estado, pues se le achacan a este las causas de los problemas en las diversas dimensiones de la gobernabilidad (Figura 3).

Acá se corrobora el planteamiento de Roitman (2001), a saber: la condición de gobernabilidad (o ingobernabilidad) siempre viene asignada por la forma de Estado que le da sentido a la perspectiva del autor, con un carácter y finalidad específicos.

El concepto de la gobernabilidad se ve cruzado por la relación de tres dimensiones que aparecen en los discursos: eficacia/eficiencia, legitimidad y estabilidad, las cuales permiten que el autor la asocie y la relacione con fenómenos de la realidad muy diversos, como los que se exponen en la Figura 2. Además, esos mismos fenómenos son los que (desde la perspectiva del autor) se deben subsanar si se quiere que el Gobierno avance en la transformación del Estado y la aplicación de sus políticas, a la vez que constituyen áreas centrales de transformación, como se expone en Soto (2014).

En la disposición de las causas de la gobernabilidad, se aprecian concomitancias con los problemas que condicionan y limitan esa forma de Estado (el ser). Este aspecto no es casual, pues como se planteó, de esta manera la transformación del Estado pasa a ser un asunto para mejorar los márgenes de gobernabilidad, no así una transformación profunda de la política y de lo político.

Los discursos son una forma de posicionar ideologías en la realidad, pero la idea misma de la ingobernabilidad es un discurso instituyente del orden, con el cual se quiere generar el escenario de relaciones para efectuar las reformas estructurales que el Gobierno pretende. Lo antedicho se evidencia al tratar de hacer pasar la reestructuración de la forma de Estado como una simple modificación en la forma institucional de ejercicio del Gobierno, para generar mejores condiciones de gobernabilidad. Con la gobernabilidad, se 
busca orientar e instituir el conflicto y los antagonismos, fundirlos en el ámbito de la política, lo cual hace que devenga un problema técnico-político. La transformación del Estado pasa al lugar de meras decisiones del ejercicio de Gobierno; pasa a tener como objetivo el logro de gobernabilidad.

La gobernabilidad es una forma de tecnificar la discusión sobre la transformación de la forma de Estado, pues con ella se hace parecer que las implicaciones solamente remiten al punto acotado del ejercicio del Gobierno, detrás de promesas tan nobles como es la satisfacción de las necesidades de la población.

En síntesis, la gobernabilidad como concepto discursivo legitima las transformaciones en una doble vía. Por un lado, las justifica, mientras las condiciones de ese Estado (el ser) provocan problemas de gobernabilidad. $Y$ por otro, señala que ciertas condiciones relacionadas a la gobernabilidad, como es la crisis de solidaridad, dificultan la aplicación de esas medidas: por lo tanto, se requiere sacrificios y la posposición de intereses por parte de algunos sectores sociales.

De lo anterior es posible inferir que el escenario que genera ese ser del Estado es de ingobernabilidad, mientras las transformaciones instituyentes del deber ser ensanchan los márgenes de la gobernabilidad, es decir, la mejoran (Figura 3).

\section{Conclusiones}

En este apartado, se exponen los principales hallazgos de la investigación realizada:

- En términos generales se evidencia que la perspectiva de gobernabilidad, que aparece en los discursos, se entiende como la posibilidad que el Gobierno desarrolle las políticas que se formularon, sin que medien factores institucionales, políticos o sectoriales que las limiten.

De la condición de gobernabilidad, en los textos del discurso, se espera que favorezca el ejercicio de Gobierno y posibilite la ejecución de las políticas del Gobierno sin mediaciones ni distorsiones; además, que no exista interferencia de intereses específicos. Estas condiciones, en su mayoría, están orientadas a eliminar la confrontación y la disidencia, vaciando de sentido la naturaleza de lo político.

- La noción de gobernabilidad presente en los discursos presidenciales de Figueres Olsen, expuestos cada primero de mayo ante la Asamblea Legislativa de Costa Rica durante su mandato, juegan un papel legitimador en el desarrollo de las políticas de este Gobierno orientadas a concretar los procesos de reconversión estatal.

La gobernabilidad, en los discursos, se presenta como condición legitimadora de la transformación estatal, pues las crisis directamente relacionadas con el 
tipo de Estado (ser) acarrean esos problemas de gobernabilidad, en cuanto impiden que se pueda gobernar, así como satisfacer las necesidades de la mayoría de la población y abrirse paso en el camino de mayor bienestar. Lo anterior aparece en los discursos como la principal razón de la necesidad de transformación del Estado. La forma lineal de la relación es el ser del Estado que lleva a la ingobernabilidad, mientras que las transformaciones orientadas al deber ser del Estado ensanchan los márgenes de gobernabilidad.

Se evidencia, también, que esta estrategia de volver la transformación del Estado en un asunto de gobernabilidad es una forma de tecnificar la discusión, pues hace parecer que las transformaciones se limitan a mejorar la posibilidad de acción de Gobierno, cuando en realidad se están planteando cambios más amplios que involucran los espacios de la política y lo político.

A su vez aparece, en la solicitud de gobernabilidad, un llamado al sacrificio de algunos sectores sociales para generar la aceptación y el apoyo (legitimación) del proyecto político en sectores sociales que se oponen a este (sectores antagónicos). También esto aparece asociado al discurso milenarista presente en menciones de un bienestar futuro por medio de la realización de concesiones y de sacrificios actuales.

Esas relaciones en los discursos validan la concepción que planteaba la gobernabilidad como un concepto-discurso orientado a la constitución de un proyecto hegemónico que, desde una visión ideológica especifica de la realidad, fomenta el desarrollo de condiciones para la materialización de un Estado especifico. Igualmente, es consecuente con la perspectiva que expone a los discursos como una herramienta de construcción de poder, pues para el contexto fungen como una práctica hegemónica orientada a la validación del proyecto político que intenta consolidar una forma determinada de Estado.

Finalmente, como otro de los aportes de la investigación, se propone la siguiente línea problemática de estudio. En el escenario de la política nacional, es común escuchar el constante empleo del discurso de la 6. Se hace referencia al gobernabilidad como por ejemplo en la realización de un "Acuerdo de acuerdo, en el año 2012, entre gobernabilidad parlamentaria" ${ }^{6}$ o la "Comisión Presidencial sobre Gobernabilidad Democrática"7. La mayoría de las veces la gobernabilidad está relacionada con transformaciones y cambios en la institucionalidad; por lo tanto se debe reflexionar sobre los usos que se le da, en el sentido de justificar acciones o de poner en un plano técnico las decisiones con sentido político que no se rigen por la eficiencia si no por el bien común.

\section{Bibliografía}

Camou, Antonio (comp.). 2001. Los desafíos de la gobernabilidad. ( $1^{\circ}$ de.). FLACSO-IISUNAM. México: Editorial Plaza y Valdés. S.A. México.

las bancadas del Partido

Liberación Nacional (PLN) y el

Partido Accesibilidad sin

Exclusión (PASE) en la

Asamblea Legislativa.

7. En referencia a la

denominada comisión de seis de notables que conformó la presidenta Laura Chinchilla, en el año 2012, para que realizaran una propuesta para fortalecer la funcionalidad y la calidad de la democracia costarricense y que en febrero de ese año (2013) presentaron su informe con una serie de

lineamientos para mejorar la condición de la democracia del país. 
Figueres, José María. 1995. Discurso del primero de mayo ante la Asamblea Legislativa de Costa Rica. Archivo de actas del plenario, Asamblea Legislativa.

Figueres, José María. 1996. Discurso del primero de mayo ante la Asamblea Legislativa de Costa Rica. Archivo de actas del plenario, Asamblea Legislativa.

Figueres, José María. 1997. Discurso del primero de mayo ante la Asamblea Legislativa de Costa Rica. Archivo de actas del plenario, Asamblea Legislativa.

Figueres, José María. 1998. Discurso del primero de mayo ante la Asamblea Legislativa de Costa Rica. Archivo de actas del plenario, Asamblea Legislativa.

Harauda, E. 2009. «Algunas aclaraciones sobre el "modelo" argumentativo de Toulmin». Contactos. 7(3): 45-56. Recuperado octubre 2, 2010, de http://www.izt.uam.mx/newpage/contactos/anterior/n73ne/toulmin.pdf

Hernández, G. Junio 1996. «El discurso del pacto Figueres-Calderón.» Revista de Ciencias Sociales. 72: 29-43. Recuperado julio 24, 2011, de http://163.178.170.74/wp-content/revistas/72/hernandez.pdf

Jäger, Sigfried. 2003. «Discurso y conocimiento: aspectos teóricos y metodológicos de la crítica del discurso y el análisis de dispositivos» En Wodak, R. y Meyer, M. (Compiladores). Métodos de análisis crítico del discurso. Barcelona: Editorial Gedisa, S.A. Barcelona, España.

Laclau, Ernesto y Chantal Mouffe. 1987. Hegemonía y estrategia socialista: Hacia una radicalización de la democracia. España: Editorial Siglo XXI.

Lukács, Georg. 1969. Historia y conciencia de clase: Estudio de dialéctica marxista. La Habana: Editorial de Ciencias Sociales. La Habana, Cuba.

O’Donnell, Guillermo. Enero 1994. Delegative Democracy, Journal of Democracy. 5 (1): 55-69. 
Ordóñez, Jaime. (Editor). 2009. ¿Hacia dónde va Costa Rica? Sistema político y escenarios de gobernabilidad democrática para la próxima década 2010-2020: 5 debates sobre el futuro de Costa Rica. (1ed.). San José, CR. Asociación Estudios para el Futuro.

Roitman, Marcos. 2001. Las razones de la democracia. ( $2^{\circ}$ ed.). Madrid España: Ediciones Sequitur. Madrid España.

Soto Kiewit, Luis Diego. 2014. «Concepción de Estado en los discursos de Figueres Olsen: un modelo genérico de reforma». Revista Rupturas. 4(2): 122-149. Disponible en: http://investiga.uned.ac.cr/revistas/index.php/rupturas/article/view/355/2 $\underline{57}$

Suárez Casanova, Alejandro. 2002. «Gobernabilidad: algunos enfoques, aproximaciones y debates actuales». Disponible en: http://unpan1.un.org/intradoc/groups/public/documents/CLAD/clad0043 431.pdf.

Tomassini, Luciano. 1996. Gobernabilidad y políticas públicas en América Latina. BID. Washington, D.C. Disponible en: http://unjobs.org/authors/luciano-tomassini.

Toulmin, Stephen. 2003. The uses of argument. New York: Cambridge University Press.

Van Dijk, Teun. (Compilador). 2005. El discurso como interacción social. Estudios sobre el discurso II. Una introducción multidisciplinaria. Barcelona: Editorial Gedisa. 